\title{
SISTEMA SILAR PARA EL CRECIMIENTO DE PELÍCULAS SEMICONDUCTORAS CON DIFERENTES APLICACIONES
}

\author{
SILAR SYSTEM TO GROWTH OF SEMICONDUCTOR FILMS \\ WITH DIFFERENT APPLICATIONS
}

David Alfredo Garzón Ramos ${ }^{1}$, Alfredo Martínez ${ }^{1}$ Daniel Rico ${ }^{1}$, Diego Alonso Guzmán Embús ${ }^{1}$, Carlos Vargas Hernández ${ }^{1}$

\footnotetext{
${ }^{1}$ Laboratorio de Propiedades Ópticas de los Materiales, Universidad Nacional de Colombia Sede Manizales, Campus La Nubia. Manizales - Caldas - Colombia, correo electrónico: dagarzonr@unal.edu.co,joamartínezma@unal.edu.co,daaricoay@unal.edu.co,daguzmane@unal.edu.co, cvargash@unal.edu.co.
}

Recibido: Marzo 5 de 2012

Aceptado: Mayo 10 de 2012

*Correspondencia del autor: Laboratorio de Propiedades Ópticas de los Materiales, Universidad Nacional de Colombia Sede Manizales, Campus La Nubia. Manizales - Caldas - Colombia, correo electrónico: dagarzonr@unal.edu.co

\section{RESUMEN}

Se diseñó e implementó un sistema electromecánico controlado por teclado que permite controlar los parámetros de crecimiento de películas semiconductoras tales como $\mathrm{ZnO}$ y $\mathrm{ZnSe}$ sobre substratos de vidrio. Los parámetros que se pueden controlar vía teclado son tiempo y secuencia de inmersión en las soluciones, además del número de ciclos. El Sistema SILAR es autónomo, versátil y cuenta con medidas de control como la temperatura. Se han empleado microcontroladores que se adaptan al sistema electromecánico y motores paso a paso que ubican los portamuestras en las soluciones respectivas, la información de los parámetros de control es visualizada en un display.

Los resultados obtenidos en los crecimientos de las películas semiconductoras indican que el sistema SILAR automatizado posee un alto desempeño que permite obtener muestras homogéneas con espesores que son controlados por medio del número de ciclos. Este sistema permite reducir los costos, el tiempo y obtener un mejor control sobre los parámetros empleados en diferentes aplicaciones.

Palabras clave: Sistema Semi-acoplado, SILAR automatizado, Películas de ZnO.

\begin{abstract}
It was designed and implemented an electromechanical system controlled by keyboard for controlling the growth parameters of semiconductor films such as $\mathrm{ZnO}$ and $\mathrm{ZnSe}$ on glass substrates. The parameters that can be controlled by keyboard are time and sequence of the immersion in the solutions, and the number of cycles. The SILAR system is autonomous, versatile, with control measures such as temperature. Microcontrollers have been used to fit the electromechanical system and stepper motors place the sample holder in the respective solutions, the information of the control parameters is displayed on a display.

The results obtained in the growth of semiconductor films indicate that the automated system SILAR has a high performance for obtaining a homogeneous sample with thicknesses controlled by the number of cycles. This system reduces costs, time and gets better control over the parameters used in different applications.
\end{abstract}

Key words: Semi-coupled system, automated SILAR, ZnO films. 


\section{INTRODUCCIÓN}

Las películas delgadas son recubrimientos de muy bajo espesor que poseen propiedades, tales como; eléctricas (fotoconductoras fotovoltaicas, rectificadoras), ópticas, magnéticas y estructurales de enorme interés investigativo, que debido a las características de las películas como grosor, morfología, esfuerzos, composición y otros, sus propiedades cambian y por tanto su campo de aplicaciones, tales como en recubrimientos, sensores de gas (1) o circuitos integrados (2).

Existen muchas metodologías de crecimiento de películas delgadas como lo son: magnetrón sputtering (3), pulsed laser ablation (4), chemical vapor deposition (5), chemical bath deposition (6), molecular beam epitaxy (7), técnicas que requieren condiciones muy especiales de crecimiento, grandes inversiones en costos, haciéndolas bastante laboriosas para aplicaciones a nivel industrial. La técnica de deposición química, método SILAR (successive ion layer adsorption and reaction), es mucho más sencillo, de bajo costo y que puede obtener resultados de alta calidad como los métodos más elaborados.

De forma sencilla, el método SILAR se basa en la inmersión de un substrato en diferentes soluciones de precursores catiónicos y aniónicos. De esa manera la técnica de deposición SILAR consiste en un ciclo de adsorción de iones, lavado y reacción.

La impurificación de las películas es un punto clave para el cambio de las propiedades. Películas delgadas de $\mathrm{ZnO}$ impurificadas con Aluminio (8) o con un post tratamiento térmico (9) hacen que sus propiedades ópticas, eléctricas y estructurales cambien significativamente, objetivo que se puede realizar con el método SILAR de una forma sencilla. Se ha encontrado que se pueden crecer películas con superficie nanoestructurada, cambiando sus propiedades de humectabilidad por la mayor área de contacto de las películas (10).

La importancia del método SILAR radica en el control de las características de crecimiento (grosor, composición, morfología y demás), a partir de la variación de los parámetros de esta técnica como lo son el tiempo de inmersión, calidad de las soluciones precursoras, temperaturas de crecimiento, $\mathrm{pH}$, numero de ciclos y concentración de iones, parámetros que son mucho más fáciles de controlar si el proceso se automatiza, facilitando aún más el control del crecimiento.

\section{MATERIALES Y MÉTODOS}

El esquema mostrado en la figura 1 ilustra la metodología de desarrollo utilizada para elaborar el sistema SILAR

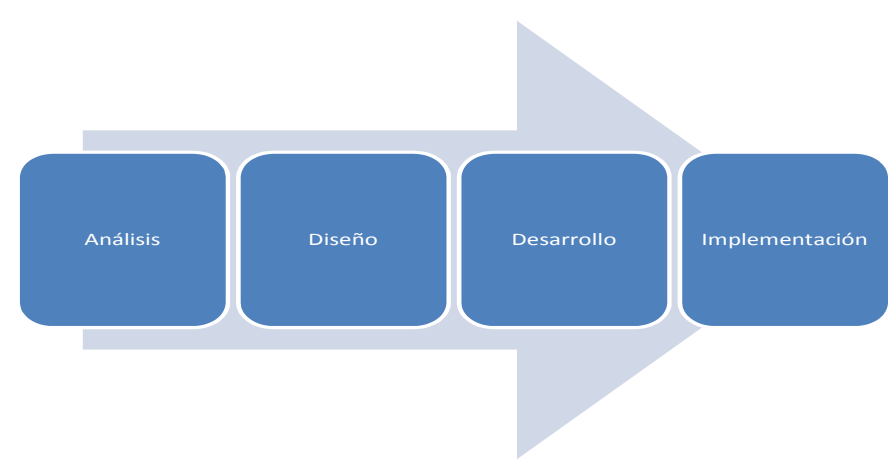

Figura 1. Metodología de desarrollo utilizada para elaborar el sistema SILAR.

\section{Análisis}

Implementando principios de ingeniería de requerimientos (11), se identificó que para cumplir con los parámetros de la técnica de crecimiento de películas SILAR, el dispositivo debe realizar inmersiones en cuatro recipientes, con un orden, número de ciclos y tiempo de inmersión que puedan ser ingresados mediante un teclado numérico, además a través de un display se deben visualizar los datos ingresados y monitorear las variables de interés (ciclos, temperatura, orden y tiempo de inmersión).

\section{Diseño}

Utilizando una metodología de desarrollo por prototipos, se han generado cinco diseños, la principal ventaja de esta metodología de desarrollo, es la capacidad de dividir el sistema en módulos, que poseen la propiedad de estar semi-acoplados conforme cumplan los requerimientos funcionales.

Estructurando así el dispositivo SILAR con un subsistema de alimentación encargado de suministrar, convertir y adaptar la energía eléctrica necesaria para ponerlo en funcionamiento, además, se cuenta con una etapa de censado conformada por una termocupla tipo J, un sensor de temperatura $\operatorname{lm} 35$ y un arreglo de amplificadores de instrumentación y cero - spam.

El subsistema encargado del control y procesamiento de datos está constituido por el micro controlador PIC18f4550 que también es responsable del funcionamiento de la interfaz de usuario integrada por un teclado numérico y un display. 
Estos cuatro subsistemas pueden ser integrados eficazmente mediante una arquitectura MVC (12) (Vista Modelo - Control).

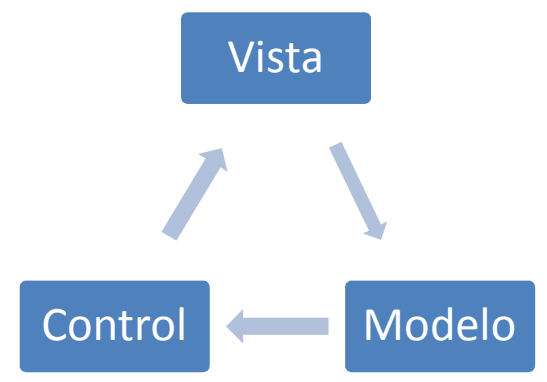

Figura 2. Arquitectura MVC.

El componente vista de la arquitectura corresponde al subsistema de interfaz de usuario, a través del cual se ingresan y muestran los parámetros de funcionamiento del sistema.

El modelo está representado por el software y firmware encargado de controlar la adquisición y conversión de señales de entrada por parte de los sensores y dispositivos I/O. En la figura 3, se muestra un diagrama de flujo donde se ilustra el modelo general de funcionamiento a seguir.

Y por último, la unidad de control, conformada por el subsistema de control y procesamiento en el cual se encuentra almacenado el modelo de funcionamiento del sistema que se encarga de que una vez sean digitados los datos de entrada y conforme determine el modelo, las señales necesarias para ubicar la muestra sobre el recipiente indicado sean emitidas.

\section{Desarrollo}

El mayor desafío evidenciado durante esta etapa fue la búsqueda de componentes que permitieran obtener el menor costo posible en el desarrollo de cada uno de los prototipos, para cumplir con este objetivo se hace uso de programas de simulación de circuitos como Proteus y CCS.

Se desarrollaron 5 prototipos de los cuales se obtuvo respectivamente un subsistema, al estar semi-acoplados cada uno de los módulos del sistema es posible realizar tareas de actualización, mejora, rediseño y monitoreo de cada uno sin la necesidad de volver a desarrollar e implementar el sistema en su totalidad.

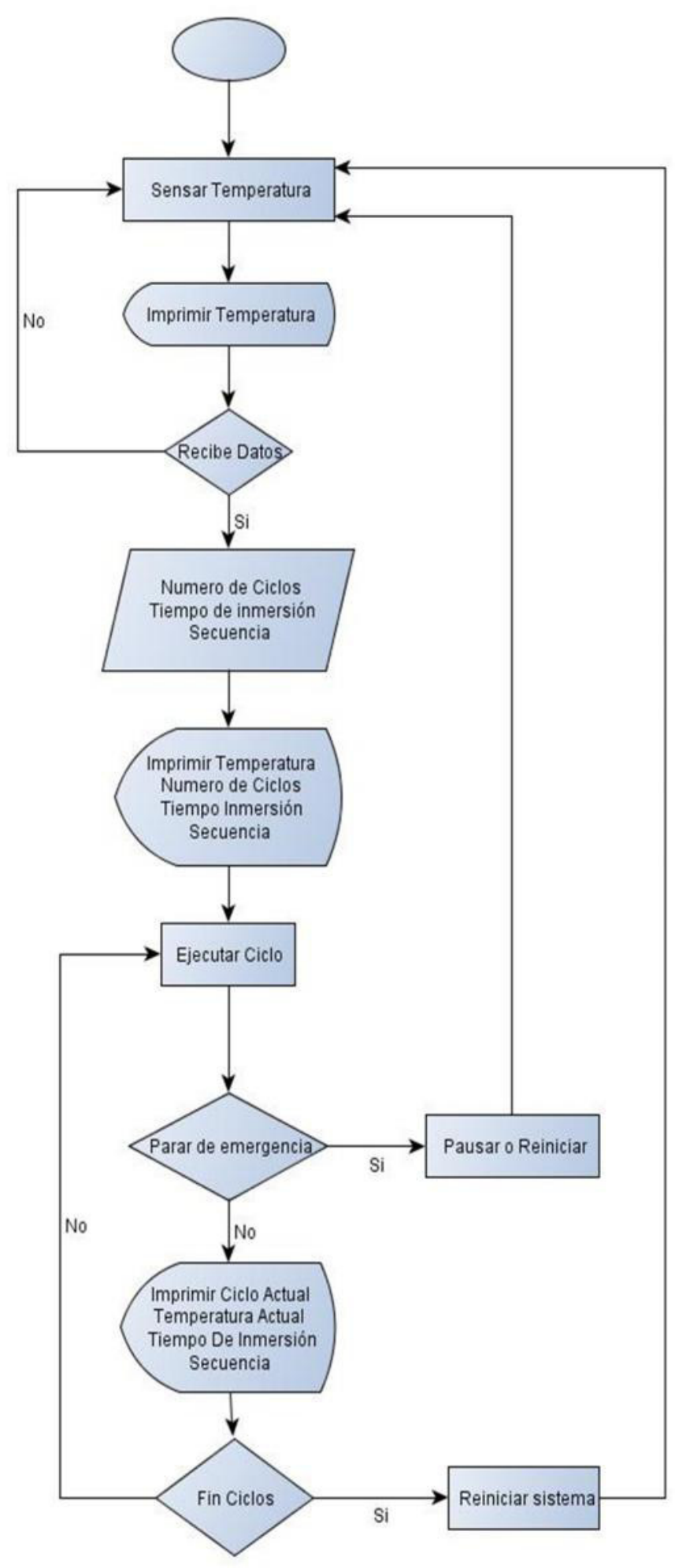

Figura 3. Diagrama general del funcionamiento a seguir. 


\section{Implementación}

En la figura 4 se puede observar el esquemático del sistema SILAR con las etapas correspondientes a alimentación, censado, control y visualización. Este esquemático fue diseñado en el ambiente de desarrollo de Proteus y luego implementado con recursos del Laboratorio de Propiedades Ópticas de los Materiales de la Universidad Nacional de Colombia sede Manizales; en la figura 5 se muestra una fotografía del sistema ya implementado.
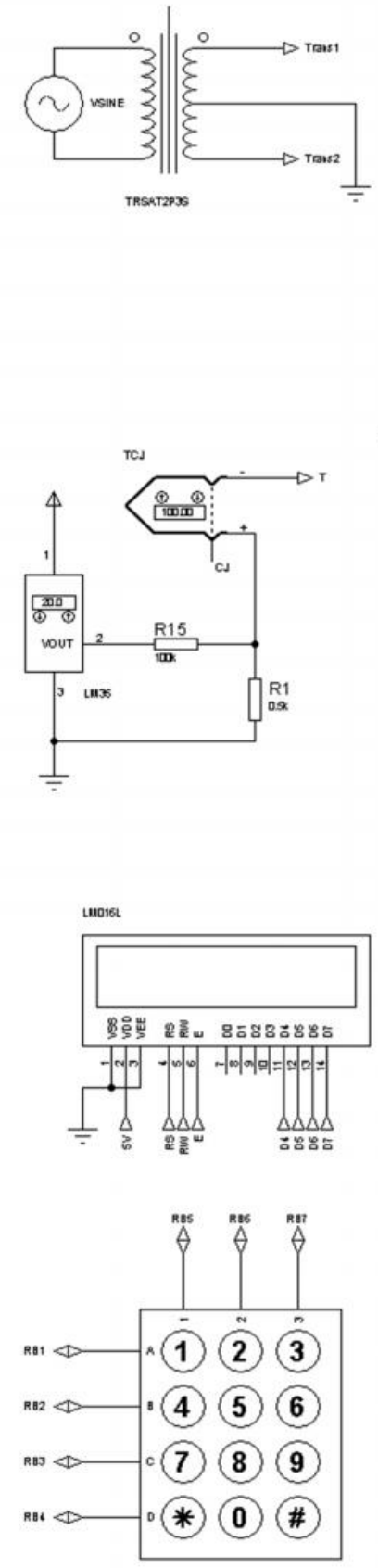
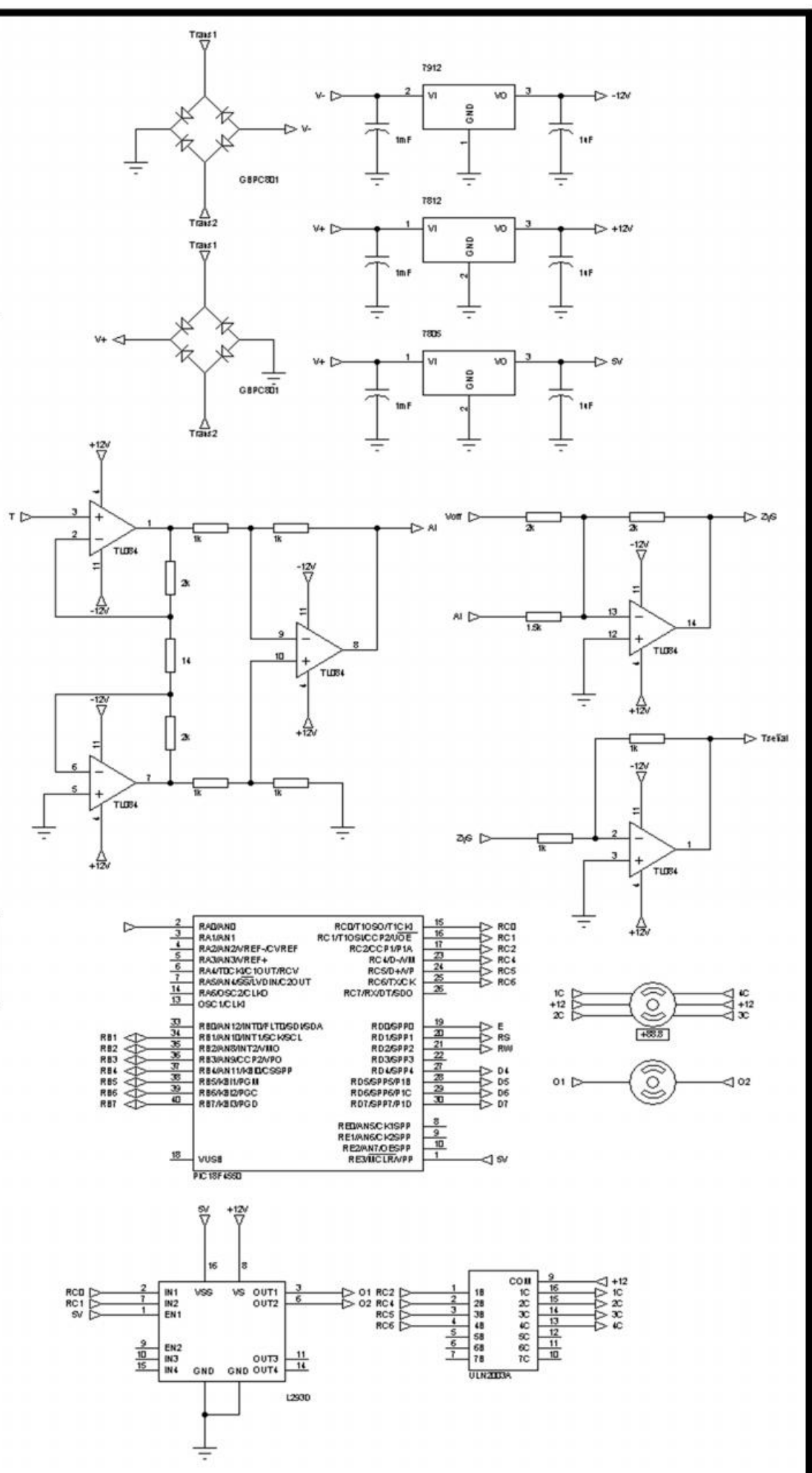

Figura 4. Esquemático del sistema SILAR. 


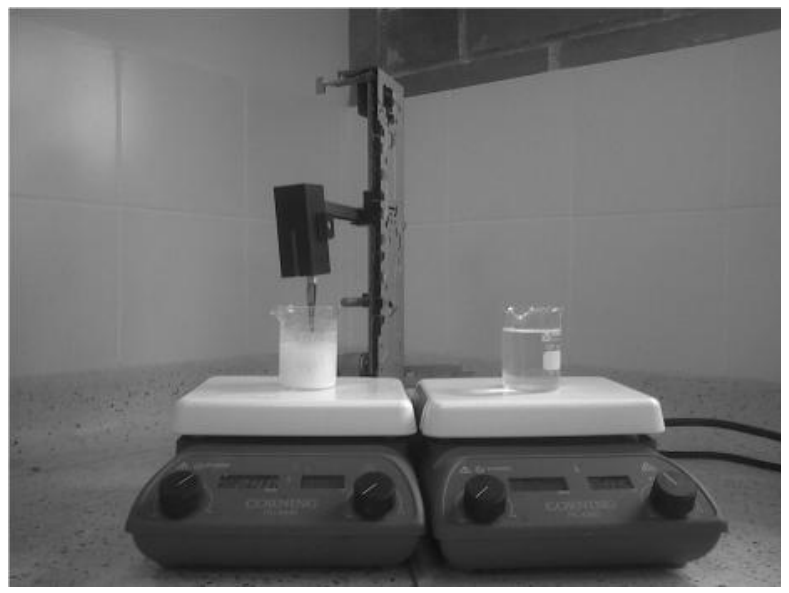

Figura 5. Prototipo implementado.

\section{RESULTADOS Y DISCUSIÓN}

Las pruebas realizadas al dispositivo "final" (Prototipo 5) evidencian el cumplimiento de cada uno de los requerimientos funcionales. Se crecieron películas sobre sustratos de vidrio tratados previamente, con una solución precursora de sulfato de zinc $(\mathrm{ZnSO} 4.7 \mathrm{H} 2 \mathrm{O})$ e hidróxido de amonio $(\mathrm{NH} 4 \mathrm{OH})$ a una relación 1:10 molar, con un lavado en agua desionizada en ebullición,

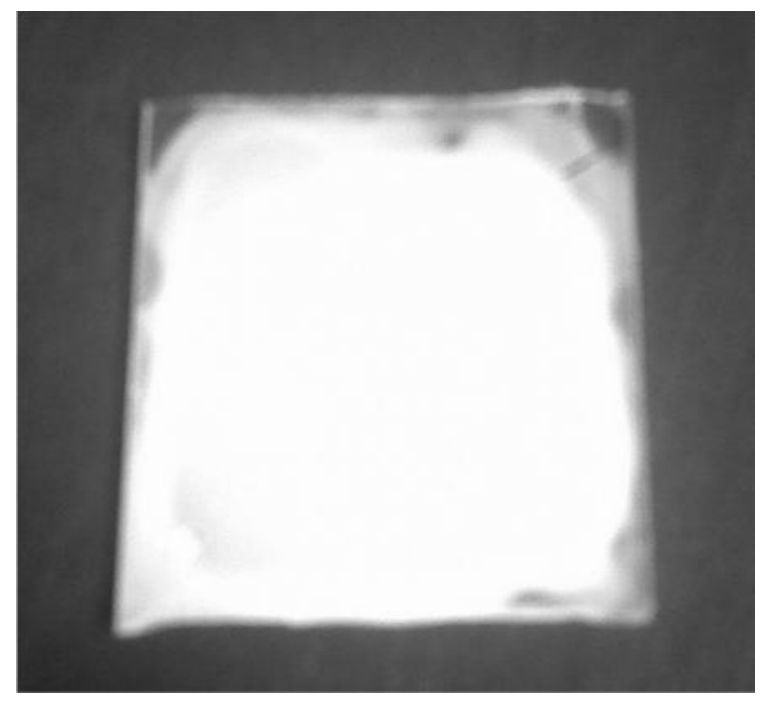

Figura 6. Recubrimiento con $\mathrm{ZnO}$ de un sustrato de vidrio a 150 ciclos. un tiempo de inmersión de dos segundos en cada solución y un numero de ciclos igual a 150. En la figura 6 se aprecia la película obtenida sobre el substrato de vidrio a 150 ciclos, además, en la figura 7 se indican los resultados del análisis de la película obtenida mediante la técnica de XRD los cuales evidencian el crecimiento de oxido de zinc ( $\mathrm{ZnO})$. La figura 8 muestra la micrografía SEM con la morfología obtenida en este procedimiento.

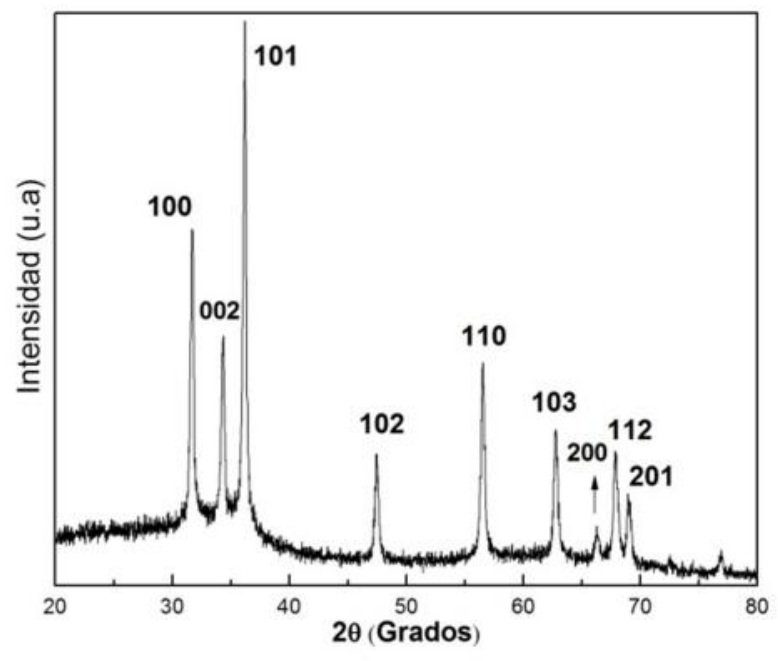

Figura 7. Patrón de XRD de la película de $\mathrm{ZnO}$ obtenida a 150 ciclos. Los índices de Miller se indican en cada pico. 


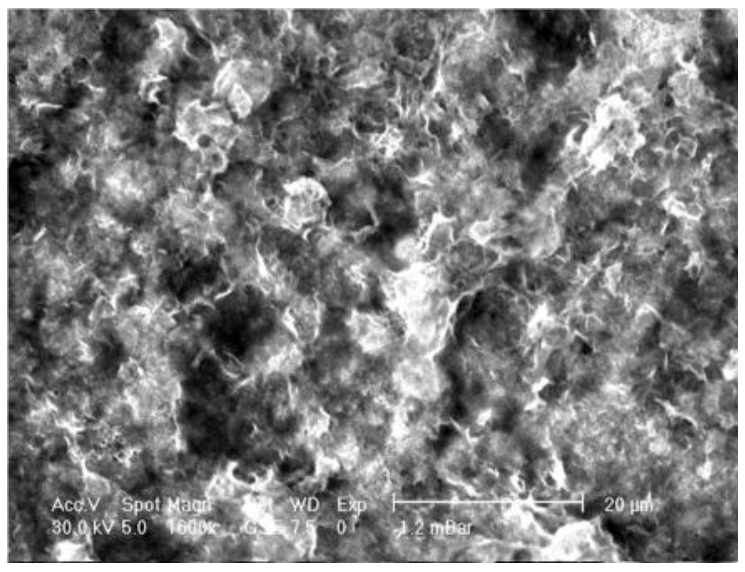

Figura 6. Micrografía SEM de la película de $\mathrm{ZnO}$ a 150 ciclos, mostrando ausencia de una morfología determinada.

\section{CONCLUSIONES}

Se optimizó la técnica de producción de películas semiconductoras mediante el desarrollo de un sistema electromecánico que permite controlar el tiempo de inmersión de la muestra, la secuencia en la que se debe ser sumergida en los porta muestras y el número de ciclos que debe ser ejecutado el proceso.
Se logró reducir considerablemente el costo de producción de películas semiconductoras comparado con los dispositivos encontrados en el mercado o con otras técnicas.

Se innovó en el campo de instrumentación física, al desarrollar una herramienta para asistir al investigador en el desarrollo de materiales

\section{AGRADECIMIENTOS}

Los autores agradecen al Ingeniero Diego Hernando Otálora y al estudiante de Ingeniería Electrónica Cristian Camilo Ceballes por su colaboración en el desarrollo del proyecto 


\section{BIBLIOGRAFÍA}

1. Li, C., Zhang, S., Hu, M. y Xie, C. Nanostructural ZnO based coplanar gas sensor arrays from the injection of metal chloride solutions: Device processing, gas-sensing properties and selectivity in liquors applications. Sensors and Actuators B, 2011; 153(2), 415-420.

2. Sun, B. and Sirringhaus, H. Solution-processed zinc oxide field effect transistors based on self-assembly of colloidal nanorods. Nano Letters. 2005; 5(12), 2408-2413.

3. Arnell, R.D., Kelly, P.J., Bradley, J.W. Recent developments in pulsed magnetron sputtering. Surface \& Coatings Technology. 2004; (188-189), 158-163.

4. Singh, S. C., Swarnkar, R. K. y Gopa, R. Zn/ZnO core/shell nanoparticles synthesized by laser ablation in aqueous environment: Optical and structural characterization. Bulletin of Materials Science. 2010; 1(33), 21-26.

5. Ataev, B.M., Mamedov, V.V., Omaev, A.K. y Magomedov, B.A. Epitaxial ZnO films grown by RFassisted low-temperature CVD method. Materials Science in Semiconductor Processing, (6), 535537.

6. Lare, Y., Godoy, A., Cattin, L., Jondo, K., Abachi, T., Diaz, F.R., Morsli, M., Napo, K., del Valle, M.A. y Bernéde, J.C. ZnO thin films fabricated by chemical bath deposition, used as buffer layer in organic solar cells. Applied Surface Science. 2009; (255), 6615-6619.

7. $\quad$ Lee, J.W., Choi, J.H., Han, S.K., Yang, S.M., Hong, S.K. y Lee, J.Y. Effects of Zn pre-exposure temperature on the microstructures of $\mathrm{ZnO}$ films grown on $\mathrm{Si}(001)$ substrates by plasma-assisted molecular beam epitaxy. Journal of Crystal Growth. 2008; (310), 1118-1123.

8. Mondal, S., Kanta, K. P. y Mitra, P. Preparation of Al-doped ZnO (AZO) Thin Film by SILAR. Journal of Physical Sciences. 2008; (12), 221-229.

9. Jimenez-González, A., Suárez-Parra, R. (1996). "Effect of heat treatment on the properties of ZnO thin films prepared by successive ion layer adsorption and reaction (SILAR)". Journal of Crystal Growth. 1996; (167), 649-655.

10. Suresh Kumar, P., Dhayal Raj, A., Mangalaraj, D. y Nataraj, D. Hydrophobic ZnO nanostructured thin films on glass substrate by simple successive ionic layer absorption and reaction (SILAR) method. Thin Solid Films. 2010; (518), e183-e186.

11. Arias Chaves, M. La ingeniería de requerimientos y su importancia en el desarrollo de proyectos de software. InterSedes: Revista de las Sedes Regionales. 2005; (4), 10-11.

12. Arquitectura de programas MVC (2011). Dpto. Lenguajes y Sistemas Informáticos Universidad de Alicante. Consultado Junio 20, 2011, en http://rua.ua.es/dspace/bitstream/10045/16627/5/pei1011tema5.pdf 